\title{
IL-33 promotes gastrointestinal allergy in a TSLP-independent manner
}

This article has been corrected since Advance Online Publication and an erratum is also printed in this issue

\author{
H Han ${ }^{1}$, F Roan ${ }^{1,2}$, LK Johnston ${ }^{3}$, DE Smith ${ }^{4}$, PJ Bryce ${ }^{3}$ and SF Ziegler ${ }^{1,5}$
}

\begin{abstract}
Atopic dermatitis (AD) often precedes asthma and food allergy, indicating that epicutaneous sensitization to allergens may be important in the induction of allergic responses at other barrier surfaces. Thymic stromal lymphopoietin (TSLP) and interleukin (IL)-33 are two cytokines that may drive type 2 responses in the skin; both are potential targets in the treatment of allergic diseases. We tested the functional role of IL-33 and the interplay between IL-33 and TSLP in mouse models of atopic march and gastrointestinal (GI) allergy. IL-33-driven allergic disease occurred in a TSLP-independent manner. In contrast, mice lacking IL-33 signaling were protected from onset of allergic diarrhea in TSLP-driven disease. Epithelialderived IL-33 was important in this model, as specific loss of IL-33 expression in the epithelium attenuated cutaneous inflammation. Notably, the development of diarrhea following sensitization with TLSP plus antigen was ameliorated even when IL-33 was blocked after sensitization. Thus, IL-33 has an important role during early cutaneous inflammation and during challenge. These data reveal critical roles for IL-33 in the "atopic march" that leads from AD to GI allergy.
\end{abstract}

The prevalence of food allergy among children in Western countries ranges around $6 \%$ to $8 \%{ }^{1}$ There is no cure or preventative treatment for food allergy, and available medications only treat symptoms after allergic reactions occur. ${ }^{2}$ Moreover, food allergy is a leading cause of anaphylaxis and imposes substantial psychosocial impact in children, adolescents, and their families. ${ }^{3}$ Recent studies suggest that the skin may be an important site for systemic sensitization to allergens, leading to the development of allergic inflammatory responses at other sites, a phenomenon referred to as the "atopic march".,

Atopic dermatitis (AD) is often the first manifestation of the atopic march, and clinical and epidemiological studies in children have established clear positive correlations between $\mathrm{AD}$ and the risk of developing food allergy. ${ }^{4,6-8}$ In addition, there is an association between skin barrier defects and development of food allergic responses, possibly due to an increased chance of sensitization by allergens permeating the skin, bypassing oral tolerance. ${ }^{6,9}$ Epidemiologic data suggest that sensitization to peanut protein can occur in children through exposure to peanut in oils applied to inflamed skin, whereas early peanut consumption is protective against allergy development and induces tolerance. ${ }^{10}$ Furthermore, mutations within the $\mathrm{AD}$ susceptibility genes filaggrin $(\mathrm{Flg})$ and SPINK5 are also associated with an increased risk of peanut allergy. ${ }^{11-17}$ In animal models, epicutaneous allergen exposure induces allergic responses in the gastrointestinal (GI) tract after challenge with the same antigen. ${ }^{18-23}$ These observations led to the hypothesis that altered barrier function in $\mathrm{AD}$ skin might facilitate cutaneous sensitization to food antigens, bypassing oral tolerance and leading to the development of food allergies.

Recently, interleukin (IL)-33, an IL-1 family cytokine, has emerged as an important initiator of allergic inflammation. ${ }^{24-26}$ ST2, the IL-33 receptor, is upregulated in the lesional skin of patients with $\mathrm{AD} .^{27,28} \mathrm{IL}-33$ has been detected at elevated levels by immunohistochemistry in eosinophilic esophagitis biopsies compared with normal esophageal tissue. ${ }^{29}$ In addition to driving Th2-type inflammation, IL-33 may also affect barrier function within the skin by downregulating filaggrin in

${ }^{1}$ Immunology Program, Benaroya Research Institute, Seattle, Washington, USA. ${ }^{2}$ Division of Allergy and Infectious Diseases, University of Washington School of Medicine, Seattle, Washington, USA. ${ }^{3}$ Division of Allergy-Immunology, Feinberg School of Medicine, Northwestern University, Chicago, Illinois, USA. ${ }^{4}$ Department of Inflammation Research, Amgen Inc., Seattle, Washington, USA and ${ }^{5}$ Department of Immunology, University of Washington School of Medicine, Seattle, Washington, USA. Correspondence: SF Ziegler (sziegler@benaroyaresearch.org) 
a
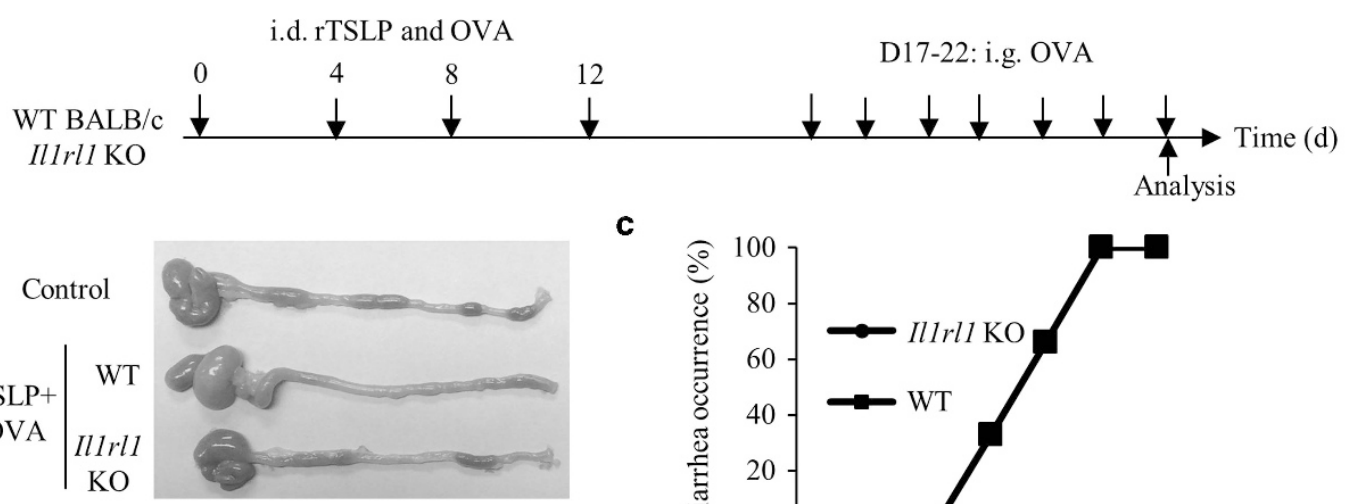

\section{b}
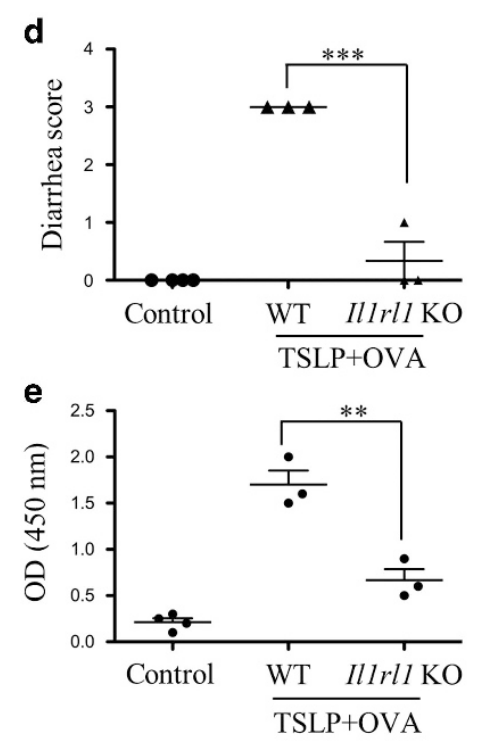

C
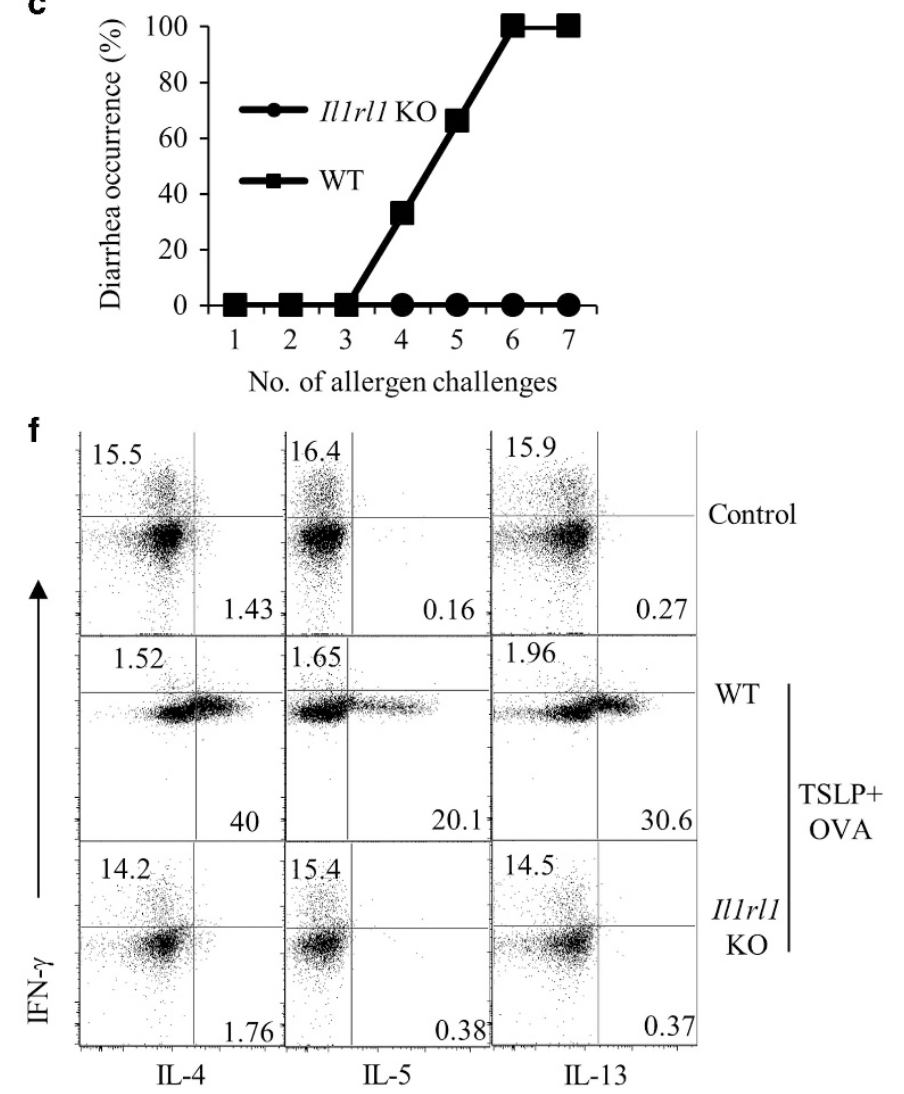

Figure 1 Interleukin (IL)-33 receptor ST2 is required in thymic stromal lymphopoietin (TSLP)-mediated gastrointestinal (GI) allergy. (a) Experimental protocol. (b) Representative photograph of the cecum and colon from indicated mice. (c) Diarrhea occurrence. (d) Diarrhea score. (e) Ovalbumin (OVA)specific lgE. (f) Intracellular cytokine staining of mesenteric lymph node (MLN) cells. Plots are gated on CD4 ${ }^{+}$CD $44^{\text {hi }}$ cells. Data are representative of two independent experiments with three to four mice per group. Error bars indicate the mean \pm s.d. ${ }^{\star \star} P \leq 0.01$ and ${ }^{* \star} P \leq 0.001$.

keratinocytes. $^{30}$ In genetic studies, single-nucleotide polymorphisms in the distal promoter of the ST2 gene locus (IL1RL1) have been associated with $\mathrm{AD} .{ }^{31}$ Mouse models have extended our understanding of the requirements for IL-33 signaling in allergic diseases, showing that local delivery of IL-33 is sufficient to drive inflammation in the skin. ${ }^{32}$ Recombinant IL-33 application intranasally in mice produces changes consistent with phenotypically early eosinophilic esophagitis, ${ }^{33}$ supporting a role for IL-33 in driving eosinophilia in eosinophilic esophagitis. These studies have led us to posit that excess IL-33 activity in the skin could have a central role in the subsequent development of GI allergy.

We previously demonstrated that skin sensitization with thymic stromal lymphopoietin (TSLP) + ovalbumin (OVA) could drive GI allergy in an atopic march model. ${ }^{21}$ The current studies further explore the pathways involved in driving disease in this model and demonstrate a requirement for IL-33 both at early and late stages of disease development. Even after sensitization, therapeutic blockade of IL-33 could ameliorate disease in this model. We also show that IL-33 alone can drive the atopic march and GI allergy in a TSLP-independent manner. This study identifies IL-33 as a crucial factor that may be common to multiple Th2-initiating events in the atopic march. These data provide additional insights into the interplay between TSLP and IL-33 that suggest novel approaches to the prevention of the atopic march and to the site-specific treatment of food allergies.

\section{RESULTS}

IL-33 receptor ST2 is required in TSLP-initiated GI allergy We have previously demonstrated that mice sensitized intradermally with TSLP and antigen develop acute diarrhea following exposure of these mice to repeated oral doses of the same antigen. $^{21}$ To further explore the interplay between epithelial cell-derived TLSP and IL-33, we tested whether IL-33 signaling was required in TSLP-driven atopic march using mice genetically deficient in the IL-33 receptor (Il1rl1 knockout (KO)) (Figure 1a). Wild-type (WT) mice sensitized with intradermal TSLP developed acute diarrheal symptoms beginning at the fourth intragastric OVA challenge and $100 \%$ of mice were 
a

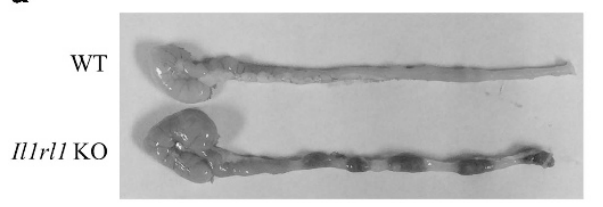

C

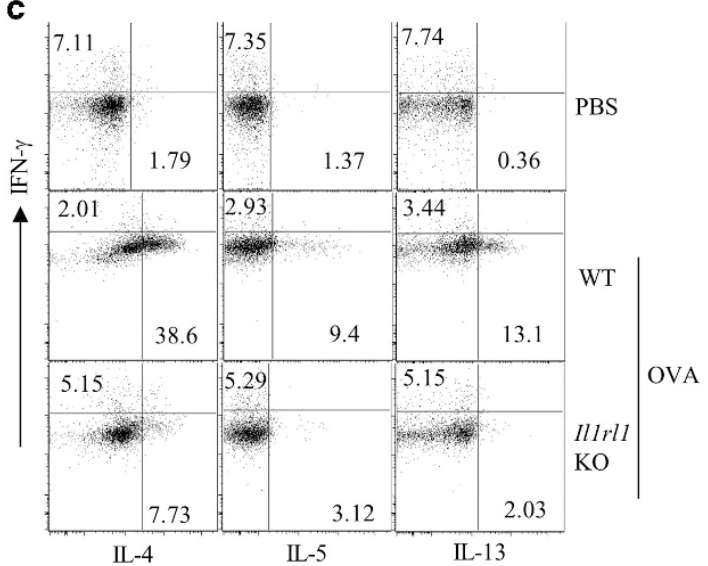

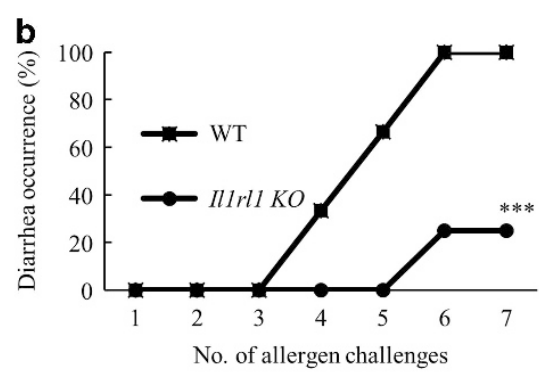

d

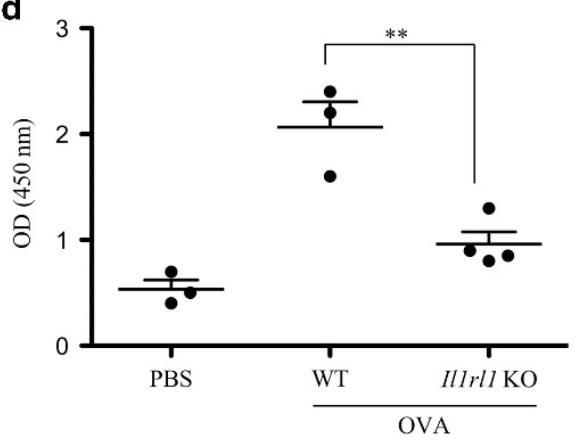

Figure 2 Gastrointestinal (GI) allergy is ST2-dependent in the ovalbumin (OVA) plus alum model. Mice were sensitized with two OVA/alum intraperitoneal injections and subsequently treated with intragastric OVA for 7 consecutive days. (a) Representative photograph of the cecum and colon from indicated mice. (b) Diarrhea occurrence. (c) Intracellular cytokine staining of mesenteric lymph node (MLN) cells isolated from mice challenged with phosphate-buffered saline (PBS) (upper) and OVA in wild-type (WT) (middle) and I/1rl1 knockout (KO) mice (lower). Plots are gated on CD4 ${ }^{+}$CD44 ${ }^{\text {hi }}$ cells. (d) OVA-specific serum IgE levels. Data are representative of two independent experiments with three to four mice per group. Error bars indicate the mean \pm s.d. ${ }^{* *} P \leq 0.01$ and ${ }^{* * *} P \leq 0.001$.

symptomatic by the sixth feed (Figure $\mathbf{1 b}-\mathbf{d}$ ). Diarrhea was also apparent through direct observation of the colon and cecum. The liquid stool observed following TSLP + OVA-induced diarrhea contrasts with the solid pellets seen in control mice. In contrast to WT mice, Il1rl1-deficient mice displayed a lack of acute diarrhea (Figure $\mathbf{1 b}-\mathbf{d}$ ) and reduced Th2 responses (Figure 1e,f) compared with Illrl1-expressing controls, demonstrating that ST2 is required in the pathogenesis of TSLP-mediated food allergy.

To expand our findings to other models of GI allergy, we next examined whether IL-33 was also required in the wellestablished OVA-alum model, ${ }^{34}$ in which mice were sensitized with OVA plus alum, followed by oral challenge with OVA for 7 consecutive days. Compared with WT mice, which developed the canonical features of allergic diarrheal disease after the fourth challenge, Illrl1-deficient mice showed significantly reduced disease onset and severity, decreased Th2 cytokine production, and lower levels of OVA-specific IgE (Figure 2a-d). These data indicate a more general role for IL-33-ST2 signaling in the development of GI manifestations of food allergy, even in the absence of priming with TSLP.

ST2 is required for TSLP-driven cutaneous inflammation To determine whether IL-33 signaling was required early during skin sensitization and/or during oral challenge in the atopic march, we compared the skin responses of WT and Illrl1-deficient mice after intradermal sensitization with TSLP + OVA (Figure 3a). Upon TSLP + OVA sensitization, control mice displayed increased cellularity in the inguinal lymph nodes, as well as elevated serum OVA-specific IgE. In contrast, inguinal lymph node cellularity, Th2 responses, and OVA-specific IgE levels in Il1rl1-deficient mice resembled that of mouse serum albumin +OVA-treated control animals (Figure 3b-e). Thus, IL-33 is required to promote TSLP-driven antigen-specific type 2 responses in the context of an AD-like skin inflammation.

Given that TSLP-mediated cutaneous inflammation requires both IL-33 production and the direct signaling of TSLP on DCs, ${ }^{21}$ we next used $\mathrm{Cd} 11 \mathrm{c}-\mathrm{Cre} \mathrm{Il3}^{\mathrm{f} / \mathrm{f}}$ mice (Supplementary Figure S1 online) to determine whether DCs were an important source of IL-33 in this model. Upon TSLP + OVA sensitization, Cd11c-

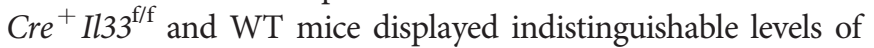
skin inflammation, as judged by elevated cellularity in skin draining lymph lodes, OVA-specific IgE levels, and Th2 cytokine production (Supplementary Figure S2). Thus, although there are requirements for both IL-33 and cell-intrinsic TSLP signaling in DCs following intradermal sensitization with TSLP + OVA, DCs are not an important source of IL-33 in the context of TSLP-driven AD-like skin inflammation.

In order to evaluate contributions of other cell populations to skin disease, we selectively deleted IL-33 in the skin epidermal keratinocytes of adult mice by Tamoxifen treatment of $\mathrm{K} 14-\mathrm{Cre}-\mathrm{ER}^{\mathrm{T2}} \mathrm{Il}-33^{\mathrm{f} / \mathrm{f}}$ mice (Supplementary Figure S3). Epidermis-specific deletion of IL-33 resulted in a dramatic attenuation of cellularity in skin draining lymph nodes, OVAspecific IgE levels, and Th2 cytokine production (Figure 4). Therefore, epidermal keratinocytes were an essential source of IL-33 in the development of this AD-like skin disease. 
a
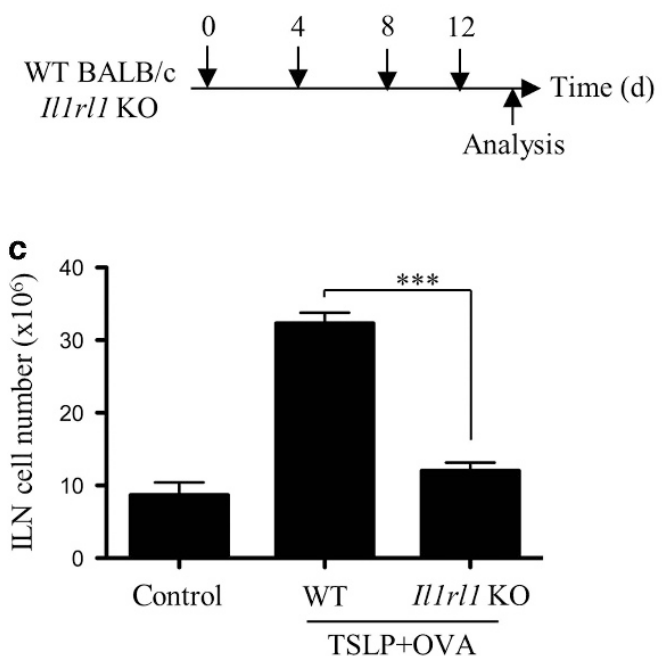

b

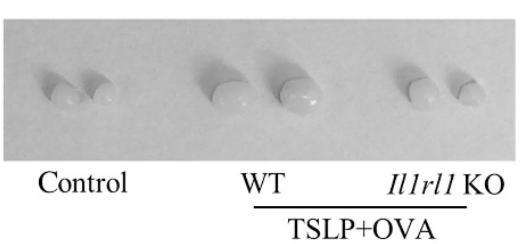

d

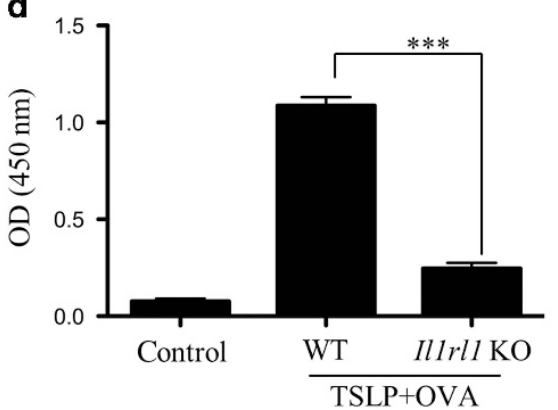

$\mathbf{e}$

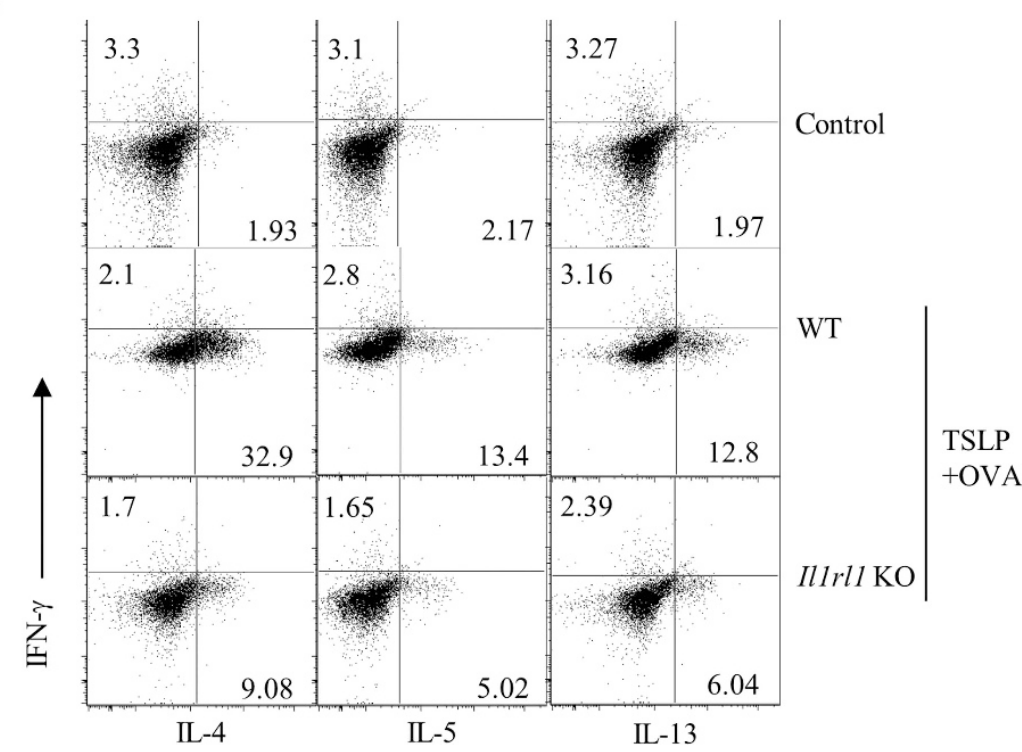

Figure 3 Interleukin (IL)-33 receptor ST2 is required for intradermal sensitization in thymic stromal lymphopoietin (TSLP)-mediated gastrointestinal (GI) allergy. (a) Experimental protocol. Mice were analyzed on day 15. (b) Representative inguinal lymph node (ILN) from mouse serum albumin (MSA) + ovalbumin (OVA) (control) and TSLP + OVA-treated mice. (c) ILN cellularity. (d) OVA-specific serum IgE levels. (e) Intracellular cytokine staining of ILN cells. Plots are gated on $\mathrm{CD} 4{ }^{+} \mathrm{CD} 44^{\text {hi }}$ cells. Data are representative of two independent experiments with three to four mice per group. Error bars indicate the mean \pm s.d. ${ }^{* *} P \leq 0.001$.

\section{Neutralization of ST2 ameliorates GI allergy}

The data presented above demonstrate a requirement for IL-33 following skin sensitization with TSLP-OVA. Our previous work has shown that TSLP-driven GI allergic disease is dependent on IL-25, but that TSLP is not required in this model after skin sensitization. ${ }^{21}$ We next tested whether IL-33 could be therapeutically targeted to prevent allergic inflammation within the gut following skin sensitization. Mice were sensitized intradermally with TSLP + OVA and then IL-33-ST2 signaling was blocked before and during repeated antigen challenge using an ST2-specific antibody $(\alpha-S T 2 \mathrm{mAb})$. Although all mice treated with a control antibody exhibited GI allergy after oral challenge, mice treated with ST2-specific neutralizing $\mathrm{mAb}$ showed decreased diarrhea development (Figure 5a-c). Flow cytometry also revealed reduced Th2 responses in anti-ST2 mAb-treated animals (Figure 5d). ST2 blockade, even after skin sensitization, ameliorated GI disease in this model. Taken together, these data suggest that the atopic march driven by TSLP is dependent on IL-33 signaling in both the skin and the gut.

\section{IL-33 is sufficient to promote antigen-induced GI allergy}

To investigate whether IL-33 alone is sufficient to promote antigen-induced GI allergy, we sensitized mice with intradermal recombinant IL-33 and OVA, followed by intragastric OVA challenge for 6 consecutive days (Figure 6a). Compared with mice treated only with phosphate-buffered saline, mice sensitized with intra dermal IL-33 developed acute diarrheal 

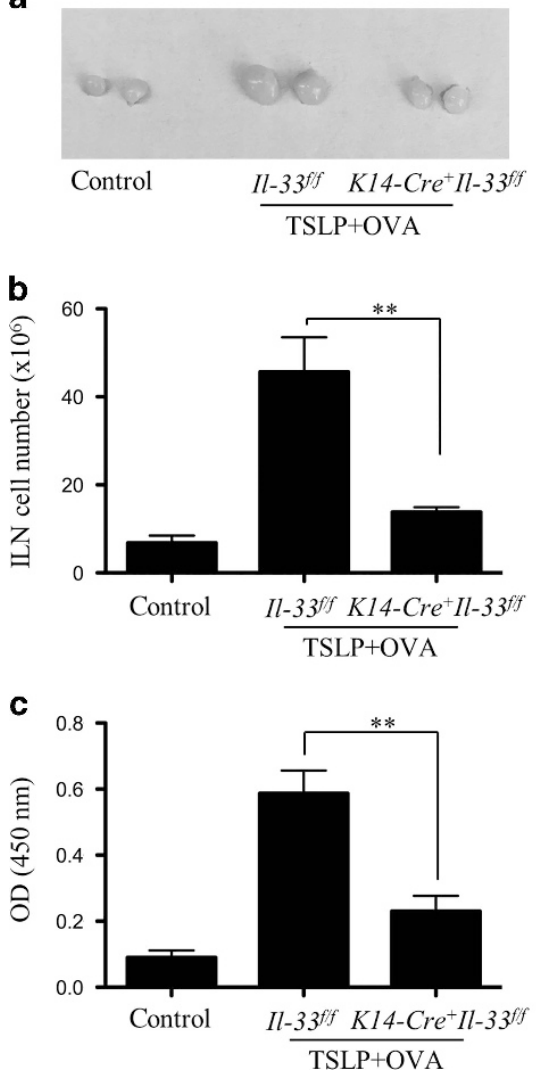

d

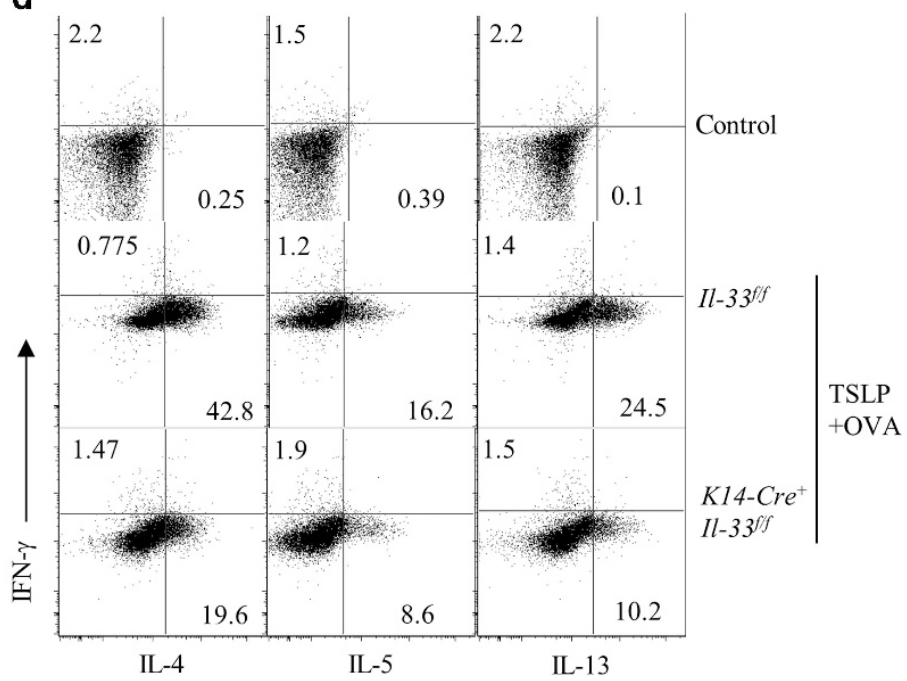
(OVA) intradermally four times and analyzed on day 15 as Figure 3. (a) Representative inguinal lymph node (ILN). (b) ILN cellularity. (c) OVA-specific serum IgE levels. (d) Cytokine production by ILN cells. Data are representative of two independent experiments with three to four mice per group. Error bars indicate the mean \pm s.d. ${ }^{\star \star} P \leq 0.01$.

symptoms beginning at the fourth administration of OVA, and $100 \%$ of mice were symptomatic by the fifth feed (Figure $\mathbf{6 b}-\mathbf{d}$ ). IL-33 + OVA-treated mice also developed other canonical features of GI allergy, such as increases in the frequency of eosinophils in peripheral blood (data not shown) and increases in serum OVA-specific IgE levels (Figure 6e). Consistent with the induction of Th2-type inflammation by IL-33 treatment, type- 2 cytokine responses were markedly elevated in IL-33 + OVA-treated mice (Figure 6f). We also observed a 60 -fold increase in mast cell-specific protease mouse MCPT-1 serum levels in mice sensitized with IL-33 + OVA versus phosphatebuffered saline + OVA, consistent with increased numbers and/or activation of mast cells (Figure 6g). Histopathological analysis of jejunum section showed that IL-33 + OVA-treated mice had a severe infiltration of eosinophils (Supplementary Figure S4). IL-33 protein in the serum was undetectable $\left(<15.6 \mathrm{pg} \mathrm{ml}^{-1}\right.$; data not shown), suggesting that circulating IL-33 is not required for the development of allergic diarrhea upon re-challenge in this model. Thus, IL-33 treatment within the skin is sufficient to promote GI allergy.

Challenge with OVA following skin administration of IL-33 + OVA elicited a robust pattern of physiologic symptoms (Figure 7a). This response was associated with a significant decrease in body temperature that was not seen in control mice (Figure $\mathbf{7 b}$ ). We next examined vascular permeability by injecting mice with Evans blue dye before the final intragastric challenge and monitoring serum leakage in the intestines. Mice sensitized with IL-33 displayed vascular leakage as demonstrated by increased Evans blue dye in the GI tract (Figure $\mathbf{7 c , d}$ ). These findings demonstrate that anaphylaxis occurs in response to oral OVA challenge after IL-33-driven skin sensitization.

\section{IL-33 drives GI allergy in a TSLP-independent manner}

To examine whether IL-33-driven disease was dependent on TSLP, we next treated TSLP receptor-deficient mice (Tslpr KO) with intra dermal OVA + IL-33, then challenged these mice with OVA by oral gavage. Tslpr KO mice responded to intra gastric OVA challenge as robustly as Tslpr-sufficient mice (Figure 8a-c), showing that IL-33 does not require TSLP signaling in this model. Although Tslpr-deficient mice displayed increased interferon- $\gamma$ production compared to controls, disease severity and Th2 responses were comparable between Tslpr-deficient and Tslpr-sufficient mice (Figure 8d-f). These data suggest that IL-33 functions independently or downstream of TSLP. 
a
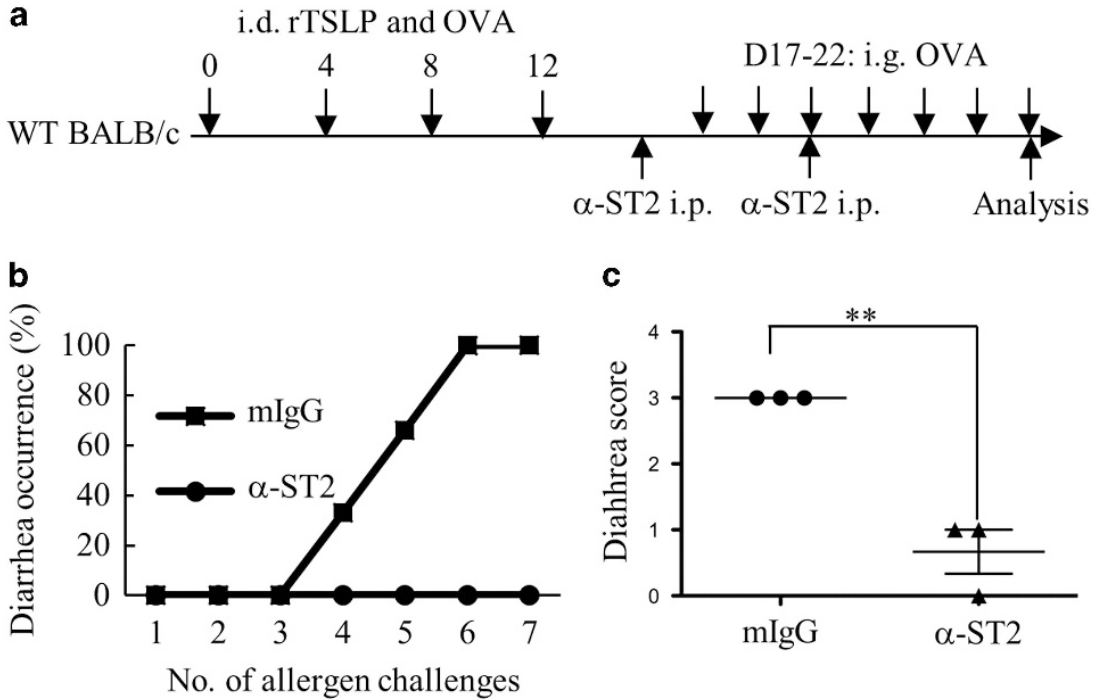

\section{C}

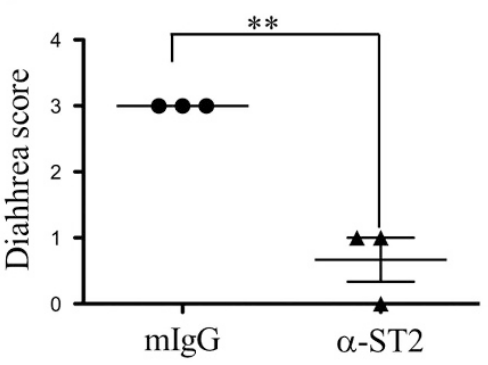

d

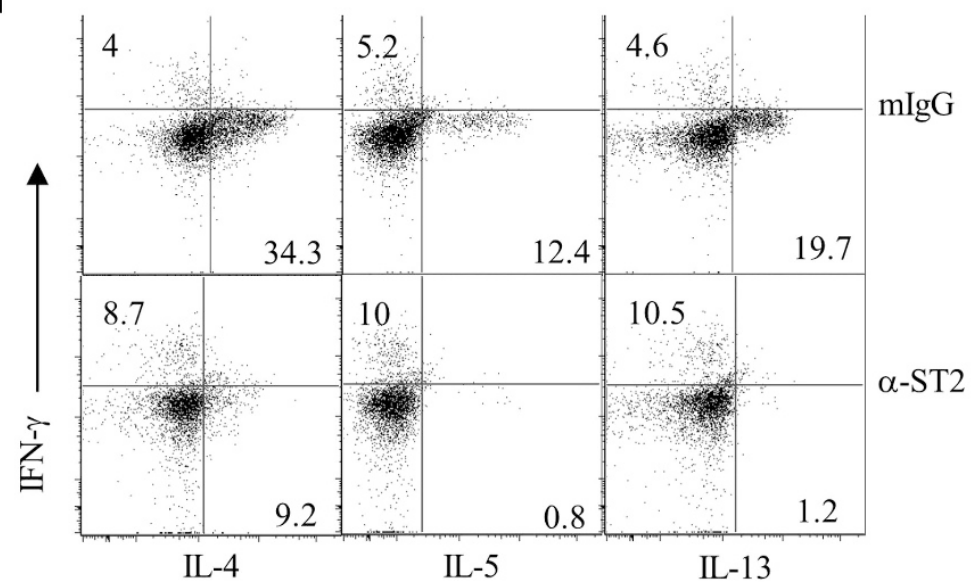

Figure 5 Neutralization of ST2 ameliorates thymic stromal lymphopoietin (TSLP)-mediated gastrointestinal (GI) allergy. (a) Experimental protocol. (b) Diarrhea occurrence. (c) Diarrhea score. (d) Intracellular cytokine staining of mesenteric lymph node (MLN) cells. Plots are gated on CD4 ${ }^{+}$CD44 ${ }^{\text {hi }}$ cells. Data are representative of two independent experiments with three mice per group. Error bars indicate the mean \pm s.d. ${ }^{\star \star} P \leq 0.01$.

\section{DISCUSSION}

$\mathrm{AD}$ often precedes the development of other atopic diseases such as asthma, allergic rhinitis, and food allergy, a phenomenon known as the "atopic march". 4 Skin barrier defects are thought to be important in both the initial local events that cause $\mathrm{AD}$ and the systemic sensitization to allergens that leads to atopic diseases at other anatomic sites. Thus, the early immunological events and factors involved in skin sensitization to allergens may be an important focal point in understanding the development of not only AD but also other atopic diseases. However, the immunological mechanisms through which antigen sensitization in the skin can predispose to allergic inflammation in the intestines and airways are incompletely understood. TSLP, IL-33, and IL-25 are three epithelial-derived cytokines that individually have been shown to have a profound influence on the development of allergic responses at barrier surfaces, although their overlapping target cell populations and inducing stimuli suggest an interplay among these cytokines to initiate, amplify, and sustain the allergic response. These three cytokines license Th2 cells in a T-cell intrinsic manner. ${ }^{35}$ Our data support the hypothesis that antigen sensitization through a disrupted skin barrier is a risk factor for the development of food allergy on oral challenge.

We have previously shown that intradermal sensitization with OVA + TSLP drives allergic inflammation within the GI tract. In this model, cutaneous sensitization elicited type 2 responses in the draining lymph nodes but not in the mesenteric lymph nodes, suggesting that systemic allergic inflammation does not occur (Supplementary Figure S5). TSLP was not required during challenge, as TSLP-deficient mice still developed disease. Loss of IL-25 signaling protected mice from diarrheal disease, demonstrating a requirement for IL-25 in TSLP-mediated GI allergy. ${ }^{21}$ We have now further characterized the cytokine pathways that function downstream of TSLP and demonstrate that IL-33 is required both for local skin inflammation following sensitization with OVA + TSLP and for diarrheal disease after oral OVA challenge in this model. Consistent with our data on the role of IL-33 in GI 
a
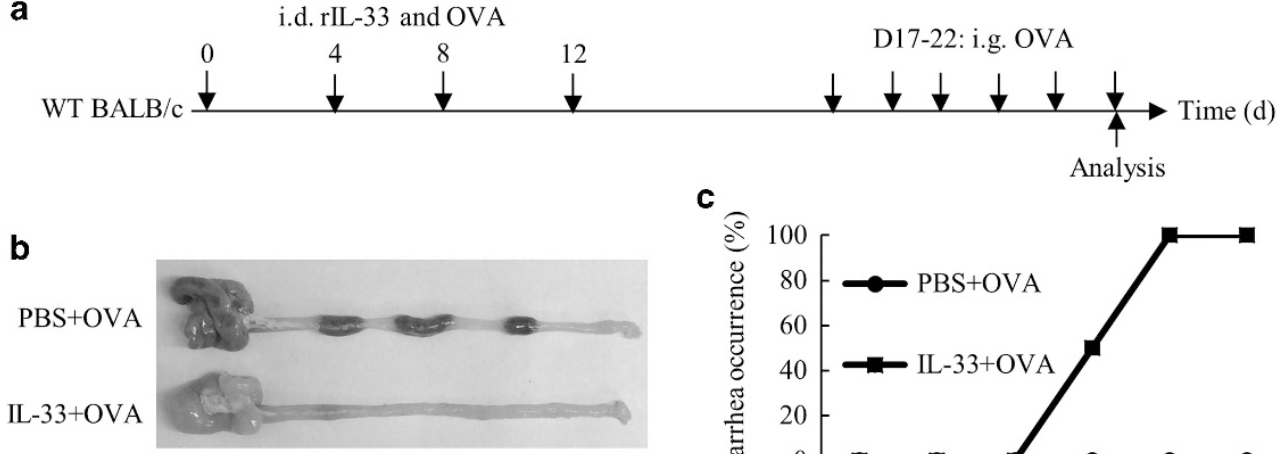

c

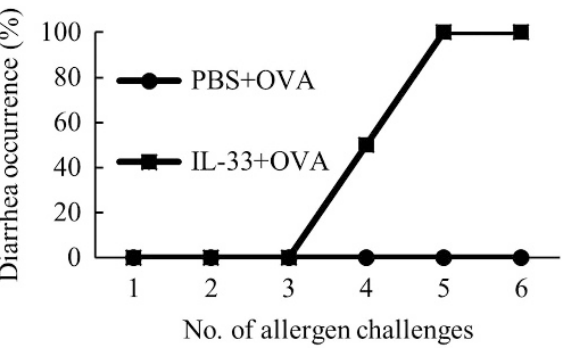

d

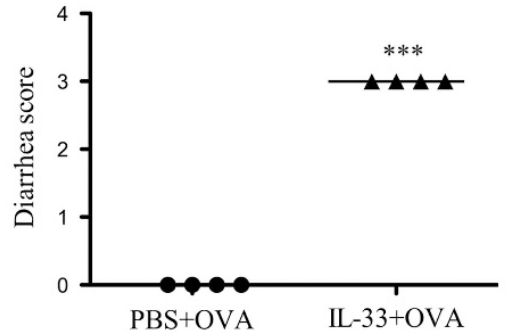

e

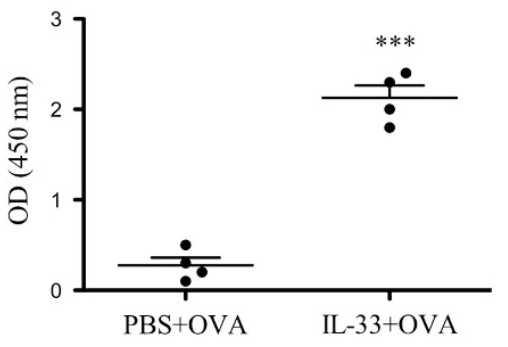

f

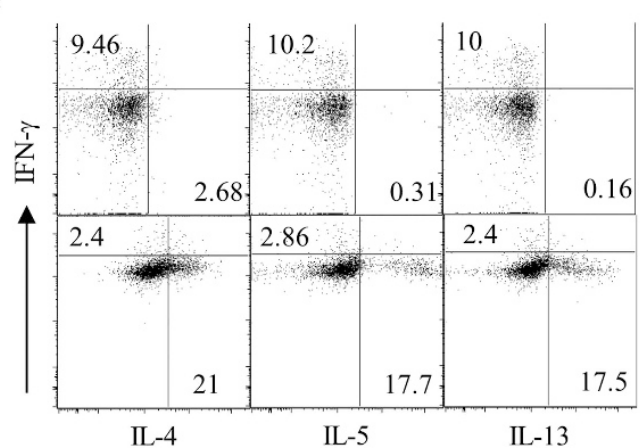

g

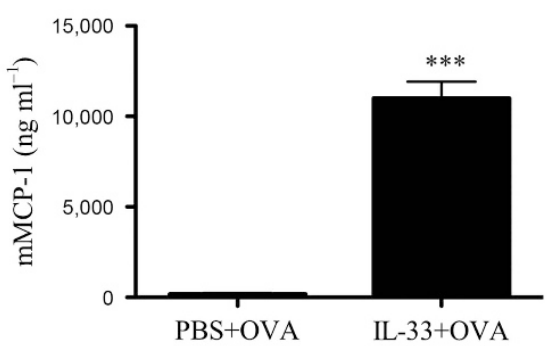

Figure 6 Intradermal administration of interleukin (IL)-33 promotes gastrointestinal (GI) allergy. (a) Experimental protocol. (b) Representative photograph of the cecum and colon from indicated mice. (c) Diarrhea occurrence. (d) Diarrhea score. (e) ovalbumin (OVA)-specific IgE. (f) Intracellular cytokine staining of mesenteric lymph node (MLN) cells isolated from mice treated with phosphate-buffered saline (PBS) + OVA (upper) and IL-33 + OVA (lower). Plots are gated on CD4 ${ }^{+} \mathrm{CD} 44^{\mathrm{hi}}$ cells. (g) Mouse MCPT-1 (mMCP-1) serum levels. Data are representative of two independent experiments with three to four mice per group. Error bars indicate the mean \pm s.d. ${ }^{\star \star \star} P \leq 0.001$.

allergy, Judd et al. ${ }^{33}$ have found elevated levels of IL-33 protein in pediatric eosinophilic esophagitis. During preparation of this manuscript, Galand et al. ${ }^{36}$ reported that IL-33 promotes IgE-mediated mast cell degranulation and food anaphylaxis. The described models provide new research tools to test new hypotheses and potential treatments in the context of food allergy.

We demonstrate that keratinocyte-derived IL-33 is crucial for promoting the allergic response during skin sensitization, as targeted deletion of IL-33 in epidermal keratinocytes, but not DCs, attenuated skin inflammation. These results raise the possibility that TSLP might directly induce IL-33 expression from keratinocytes; however, we have found that IL-33 is highly expressed constitutively within the keratinocyte layer, based on examination of reporter mice for IL-33 (Supplementary Figure S6). Additional studies will be required to determine whether IL-33 levels remain stable during inflammation, are induced by TSLP or other TSLP-induced cytokines, or are increased through IL-33 release by cells after tissue damage. Regardless of whether IL-33 levels are directly induced by TSLP, IL-33 likely has a critical role in initiating a feed-forward loop to amplify type 2 responses. This amplification may occur, in part, because TSLP induces the accumulation of many inflammatory cells that express the IL-33 receptor and respond to IL-33. Mucosal mast cells secrete prodigious amounts of IL- 9 and IL-13 in response to IL-33, which contributes to IgE antibody production. ${ }^{37}$ IL-33 has also been shown to directly stimulate eosinophil differentiation and amplify IgE synthesis. ${ }^{38,39}$ Identification of the specific cellular responses that depend on IL-33 in TSLP-driven disease will be important in our understanding of the propagation of allergic inflammation in the atopic march. 
a

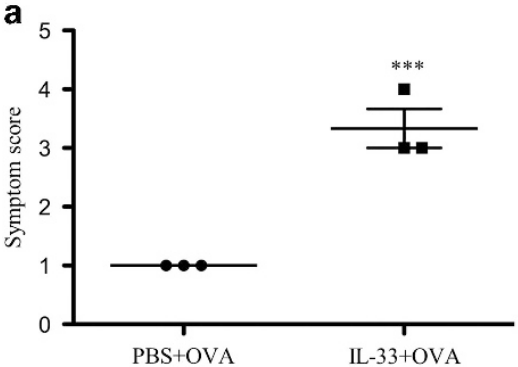

c

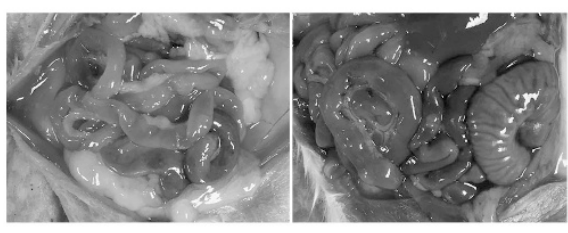

PBS + OVA

IL-33+OVA b
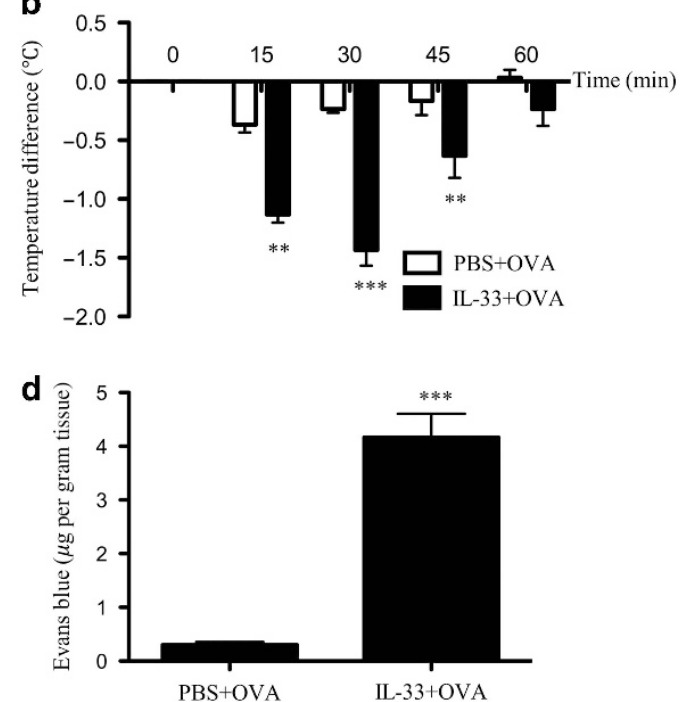

Figure 7 Antigen-driven anaphylaxis in interleukin (IL)-33 + ovalbumin (OVA)-sensitized mice. (a) Symptom scores. (b) Body temperature responses after antigen challenge. (c,d) Serum leakage at intestines. Data are representative of two independent experiments with three mice per group. Error bars indicate the mean \pm s.d. ${ }^{* *} P \leq 0.01$ and ${ }^{* * *} P \leq 0.001$.

It is notable that whereas TSLP-mediated disease required IL-33 early in the cutaneous inflammatory response as well as during oral challenge, TSLP is dispensable in an atopic march model driven by IL-33. Similar to TSLP, intradermal treatment with IL-33 + OVA drives local inflammation in the skin and results in systemic antigen sensitization and development of GI diarrheal disease after intragastric OVA challenge. Yet, IL-33mediated disease severity and induction of type 2 cytokines were unchanged in the absence of TSLP signaling. Tslpr KO mice did demonstrate increased interferon $-\gamma$ production, suggesting a role for TSLP in downregulating Th1 responses in this model. Consistent with this, interferon- $\gamma$ blockade can restore Th2-type immunity in Trichuris muris infection of Tslpr KO mice. ${ }^{40}$

Our work and others clearly reveal that IL-33, IL-25, and TSLP are differentially required to promote the atopic march. ${ }^{18-23}$ The distinct requirements for TSLP, IL-33, and IL-25 during sensitization and challenge provide a mechanistic basis for the growing clinical recognition of the heterogeneity of allergic diseases at different mucosal sites. Elucidating how these epithelial cell-derived cytokines regulate their target cell populations at different sites and stages of disease will further our understanding of the natural history of atopic diseases and the ways that blockade of specific cytokines may differentially influence disease development and progression. Our study demonstrates important roles for IL-33 signaling in both the early and late stages of the atopic march. The ability to ameliorate disease after sensitization in an atopic march and food allergy model suggests that IL-33 may be an important target in both the prevention and treatment of food allergies.

\section{MATERIALS AND METHODS}

Mice and treatments. Six- to 8-week-old female BALB/c mice were obtained from Charles River Laboratories (San Diego, CA). Illrl1-deficient mice were provided by Dr. Andrew McKenzie (Medical
Research Council Laboratory of Molecular Biology, Cambridge, UK). Details of the procedure and analysis of the resulting phenotype of conditional Il33-deficient mice (also used as IL-33 reporter) will be described elsewhere (Johnston LK and Bryce PJ, manuscript in preparation). All mice were certified to be specific pathogen free and cared for in accordance with the guidelines of the Institutional Animal Care and Use Committee at Benaroya Research Institute (Seattle, WA). Intradermal injections were performed as previously described. ${ }^{20,21}$ Briefly, $5 \mu \mathrm{g}$ TSLP (Amgen, Thousand Oaks, CA) or $2.5 \mu \mathrm{g}$ IL-33 (R\&D Systems, Minneapolis, MN, and BioLegend, San Diego, CA), or mouse serum albumin (Sigma-Aldrich, St. Louis, MO) with $2.5 \mu \mathrm{g}$ OVA (A7642; Sigma-Aldrich) were injected intradermally in a $100 \mu \mathrm{l}$ volume of sterile phosphate-buffered saline every 3 days for a total of four times. Intragastric challenges with $50 \mathrm{mg}$ of OVA (grade V, A5503; Sigma-Aldrich) were performed as described previously. ${ }^{34}$ OVA plus alum model was performed as previously described. ${ }^{34}$ Mice demonstrating profuse liquid stool were recorded as diarrhea-positive animals. A detailed clinical score was assessed up to $1 \mathrm{~h}$ after final oral feeding (0: normal; 1: soft; 2: running; 3: liquid; 4: bloody). Symptom scoring was performed after 4 th challenge as described before. ${ }^{21,41}$ For blockade with ST2-specific mAb, mice were injected with $500 \mu \mathrm{g}$ of control mouse IgG1 or muST2-specific muIgG1 mAb (Amgen) intraperitoneally on days 15 and 19. K14-Cre- $E R^{T 2}$ mice were purchased from Jackson Laboratories (Sacramento, CA). Six- to 8-week-old $\mathrm{K}_{14}-\mathrm{Cre}^{+} \mathrm{Il33} 3^{\mathrm{f} / \mathrm{f}}$ mice and littermates were injected intraperitoneally with Tamoxifen (Sigma-Aldrich, $0.3 \mathrm{mg}$ in $100 \mu \mathrm{l}$ corn oil, for 5 consecutive days) and subjected to experiments two weeks after Tamoxifen injection.

Vascular permeability measurements. Vascular permeability was evaluated by measuring the leakage of Evans blue dye into the intestine as described before. ${ }^{21,42}$

Measurement of body temperature. The changes in body temperature of mice were measured using a Dual Laser IR Thermometer (42509, Extech, Waltham, MA), at 0, 15, 30, 45, and 60 min after 5th OVA challenge as described before. ${ }^{21}$

Cell culture. Mesenteric lymph node and inguinal lymph node cells were isolated and cultured in RPMI medium with $10 \%$ fetal calf serum, penicillin, and streptomycin, with $100 \mu \mathrm{g} \mathrm{ml}^{-1}$ OVA for $48 \mathrm{~h}$. Cells were then stimulated with phorbol 12-myristate 13-acetate (PMA) and 
a

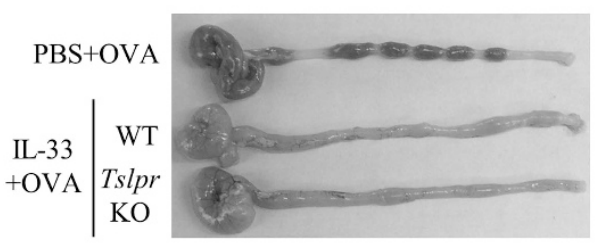

C

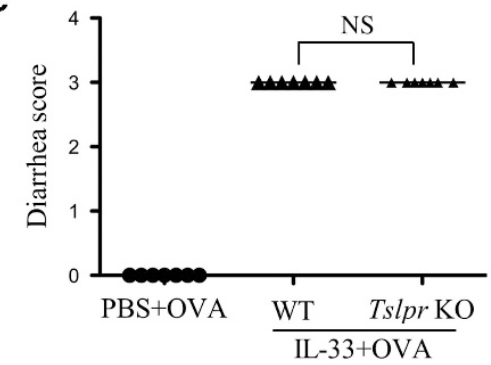

d
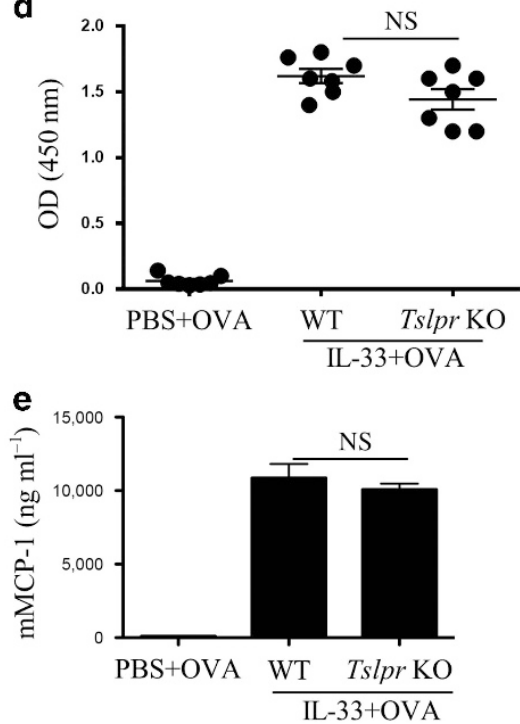

f b
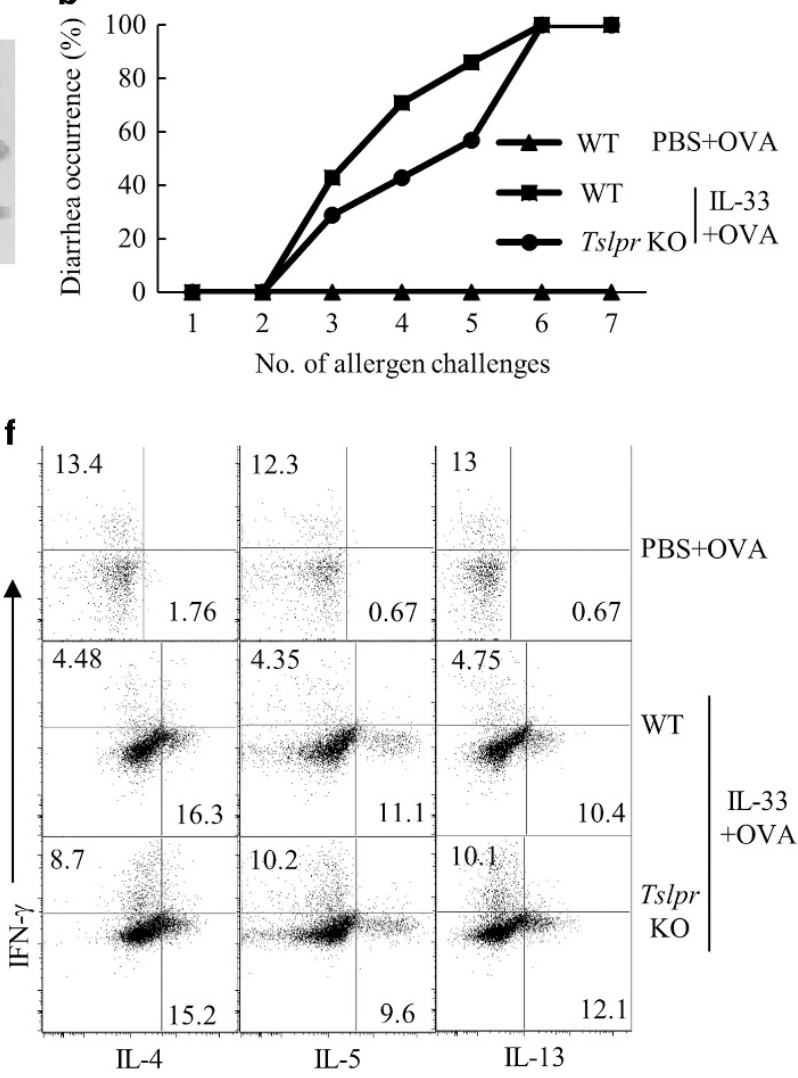

Figure 8 Interleukin (IL)-33 mediates gastrointestinal (GI) allergy in a thymic stromal lymphopoietin (TSLP)-independent manner. (a) Representative photograph of the cecum and colon from indicated mice. (b) Diarrhea occurrence. (c) Diarrhea score. (d) ovalbumin (OVA)-specific IgE. (e) Mouse MCPT-1 (mMCP-1) serum levels. Data were pooled from two independent experiments $(n=7)$. (f) Intracellular cytokine staining of MLN cells. Plots are gated on $\mathrm{CD} 4{ }^{+} \mathrm{CD} 44^{\mathrm{hi}}$ cells. Error bars indicate the mean \pm s.d.

ionomycin in the presence of brefeldin A for $4-5 \mathrm{~h}$. The cells were stained and analyzed for cytokine production by flow cytometry. Further analyses were performed using FlowJo software (Tree Star, Ashland, OR).

Enzyme-linked immunosorbent assay. Mouse MCPT-1 levels were measured by enzyme-linked immunosorbent assay (ELISA) according to the manufacturer's instruction (eBioscience, San Diego, CA). Detection of OVA-specific IgE has been described before. ${ }^{43,44}$ For $\mathrm{Cd} 11 \mathrm{c}^{\mathrm{Cre}} \mathrm{Il} 33^{\mathrm{f} / \mathrm{f}}$ mice experiment, mesenteric lymph node cells were isolated and cultured in RPMI medium with $10 \%$ fetal calf serum, penicillin, and streptomycin, with $50 \mu \mathrm{l}$ Dynabeads Mouse T-Activator CD3/CD28 (Gibco, Gaithersburg, MD) or $100 \mu \mathrm{g} \mathrm{ml}^{-1}$ OVA for $48 \mathrm{~h}$. Concentrations of IL-5 (BioLegend) and IL-13 (eBioscience) in the culture supernatants were determined by sandwich ELISA following the manufacturer's protocol.

Histology. Ears were incubated in $1 \%$ paraformaldehyde (PFA) solution followed by $30 \%$ sucrose before being frozen in embedding medium containing $7.5 \%$ gelatin and $15 \%$ sucrose in phosphate-buffered saline. Frozen tissues were sectioned and stained with 4',6-diamidino-2-phenylindole. Green fluorescent protein indicates IL-33 protein expression.

Tissue lysate ELISA. Ear tissue was excised and placed in T-PER tissue protein extraction reagent (Thermo Fisher Scientific, Waltham, MA). Tissue was homogenized and protein was quantified using a Nanodrop spectrophotometer at $280 \mathrm{~nm}$. One hundred micrograms of total protein per sample was analyzed using the R\&D Systems murine IL-33 ELISA kit following the manufacturer's protocol.

Statistics. Statistical analyses were performed with Prism software (GraphPad, La Jolla, CA). Two means were compared using a nonparametric two-tailed Mann-Whitney test. Three or more means were compared using one-way analysis of variance with a Tukey's posthoc test with significance between groups represented as ${ }^{\star} P \leq 0.05$, ${ }^{* *} P \leq 0.01$, and ${ }^{* *} P \leq 0.001$.

SUPPLEMENTARY MATERIAL is linked to the online version of the paper at http://www.nature.com/mi 


\section{ACKNOWLEDGMENTS}

We thank Dr A. McKenzie for providing //1rl1-deficient mice. We thank the members of the Ziegler laboratory for discussion. This work was partially supported by NIH grants AI068731, HL098067, Al124220, Al125378, and AR059058 to S.F.Z.

\section{AUTHOR CONTRIBUTIONS}

H.H. and S.F.Z. developed the study. H.H. designed and performed the experiments. L.K.J. and P.J.B. performed the immunohistochemistry and

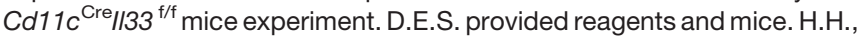
F.R. and S.F.Z. wrote the manuscript.

\section{DISCLOSURE}

The authors declared no conflict of interest.

c) 2018 Society for Mucosal Immunology

\section{REFERENCES}

1. Nowak-Wegrzyn, A. \& Sampson, H.A. Adverse reactions to foods. Med. Clin. North Am. 90, 97-127 (2006).

2. Wang, J. \& Sampson, H.A. Treatments for food allergy: how close are we? Immunol. Res. 54, 83-94 (2012).

3. Cummings, A.J., Knibb, R.C., King, R.M. \& Lucas, J.S. The psychosocial impact of food allergy and food hypersensitivity in children, adolescents and their families: a review. Allergy 65, 933-945 (2010).

4. Spergel, J.M. \& Paller, A.S. Atopic dermatitis and the atopic march. J. Allergy Clin. Immunol. 112, S118-S127 (2003).

5. Li, M. Current evidence of epidermal barrier dysfunction and thymic stromal lymphopoietin in the atopic march. Eur. Respir. Rev. 23, 292-298 (2014).

6. Lack, G. et al. Factors associated with the development of peanut allergy in childhood. N. Engl. J. Med. 348, 977-985 (2003).

7. Sicherer, S.H. \& Leung, D.Y. Advances in allergic skin disease, anaphylaxis, and hypersensitivity reactions to foods, drugs, and insects in 2013. J. Allergy Clin. Immunol. 133, 324-334 (2014).

8. Burks, A.W. et al. ICON: food allergy. J. Allergy Clin. Immunol. 129, 906-920 (2012).

9. Bieber, T. Atopic dermatitis. N. Engl. J. Med. 358, 1483-1494 (2008).

10. Du Toit, G. et al. Randomized trial of peanut consumption in infants at risk for peanut allergy. N. Engl. J. Med. 372, 803-813 (2015).

11. Walley, A.J. et al. Gene polymorphism in Netherton and common atopic disease. Nat. Genet. 29, 175-178 (2001).

12. Brown, S.J. et al. Loss-of-function variants in the filaggrin gene are a significant risk factor for peanut allergy. J. Allergy Clin. Immunol. 127, 661-667 (2011).

13. Tan, H.T. et al. Filaggrin loss-of-function mutations do not predict food allergy over and above the risk of food sensitization among infants. J. Allergy Clin. Immunol. 130, 1211-1213 e1213 (2012).

14. Kusunoki, T. et al. SPINK5 polymorphism is associated with disease severity and food allergy in children with atopic dermatitis. J. Allergy Clin. Immunol. 115, 636-638 (2005).

15. Venkataraman, D. et al. Filaggrin loss-of-function mutations are associated with food allergy in childhood and adolescence. J. Allergy Clin. Immunol. 134, 876-882 e874 (2014).

16. Brough, H.A. et al. Peanut allergy: effect of environmental peanut exposure in children with filaggrin loss-of-function mutations. J. Allergy Clin. Immunol. 134, 867-875 e861 (2014).

17. Brough, H.A. et al. Atopic dermatitis increases the effect of exposure to peanut antigen in dust on peanut sensitization and likely peanut allergy. J. Allergy Clin. Immunol. 135, 164-170 (2015).

18. Demehri, S., Morimoto, M., Holtzman, M.J. \& Kopan, R. Skin-derived TSLP triggers progression from epidermal-barrier defects to asthma. PLOS Biol. 7, e1000067 (2009).

19. Zhang, Z. et al. Thymic stromal lymphopoietin overproduced by keratinocytes in mouse skin aggravates experimental asthma. Proc. Natl Acad. Sci. USA 106, 1536-1541 (2009).
20. Han, H. et al. Thymic stromal lymphopoietin (TSLP)-mediated dermal inflammation aggravates experimental asthma. Mucosal Immunol. 5, 342-351 (2012).

21. Han, H., Thelen, T.D., Comeau, M.R. \& Ziegler, S.F. Thymic stromal lymphopoietin-mediated epicutaneous inflammation promotes acute diarrhea and anaphylaxis. J. Clin. Invest. 124, 5442-5452 (2014).

22. Noti, M. et al. Thymic stromal lymphopoietin-elicited basophil responses promote eosinophilic esophagitis. Nat. Med. 19, 1005-1013 (2013).

23. Noti, M. et al. Exposure to food allergens through inflamed skin promotes intestinal food allergy through the thymic stromal lymphopoietin-basophil axis. J. Allergy Clin. Immunol. 133, 1390-1399. 1399 e1391-1396 (2014).

24. Schmitz, J. et al. IL-33, an interleukin-1-like cytokine that signals via the IL-1 receptor-related protein ST2 and induces Thelper type 2-associated cytokines. Immunity 23, 479-490 (2005).

25. Molofsky, A.B., Savage, A.K. \& Locksley, R.M. Interleukin-33 in Tissue Homeostasis, Injury, and Inflammation. Immunity 42, 1005-1019 (2015).

26. Liew, F.Y., Girard, J.P. \& Turnquist, H.R. Interleukin-33 in health and disease. Nat. Rev. Immunol. 16, 676-689 (2016).

27. Savinko, T. et al. IL-33 and ST2 in atopic dermatitis: expression profiles and modulation by triggering factors. J. Invest. Dermatol. 132, 1392-1400 (2012).

28. Savinko, T. et al. ST2 regulates allergic airway inflammation and T-cell polarization in epicutaneously sensitized mice. J. Invest. Dermatol. 133, 2522-2529 (2013).

29. Simon, D., Radonjic-Hosli, S., Straumann, A., Yousefi, S. \& Simon, H.U. Active eosinophilic esophagitis is characterized by epithelial barrier defects and eosinophil extracellular trap formation. Allergy 70, 443-452 (2015).

30. Seltmann, J., Roesner, L.M., von Hesler, F.W., Wittmann, M. \& Werfel, T. IL-33 impacts on the skin barrier by downregulating the expression of filaggrin. J. Allergy Clin. Immunol. 135, 1659-1661 e1654 (2015).

31. Shimizu, M. et al. Functional SNPs in the distal promoter of the ST2 gene are associated with atopic dermatitis. Hum. Mol. Genet. 14, 2919-2927 (2005).

32. Imai, Y. et al. Skin-specific expression of IL-33 activates group 2 innate lymphoid cells and elicits atopic dermatitis-like inflammation in mice. Proc. Natl Acad. Sci. USA 110, 13921-13926 (2013).

33. Judd, L.M. et al. Elevated IL-33 expression is associated with paediatric eosinophilic esophagitis, and exogenous IL-33 promotes eosinophilic esophagitis development in mice. Am. J. Physiol. Gastrointest. Liver Physiol., ajpgi 00290 02015310, G13-G25 (2015).

34. Brandt, E.B. et al. Mast cells are required for experimental oral allergeninduced diarrhea. J. Clin. Invest. 112, 1666-1677 (2003).

35. Van Dyken, S.J. et al. A tissue checkpoint regulates type 2 immunity. Nat. Immunol. 17, 1381-1387 (2016).

36. Galand, C. et al. IL-33 promotes food anaphylaxis in epicutaneously sensitized mice by targeting mast cells. J. Allergy Clin. Immunol. 138, 1356-1366 (2016).

37. Chen, C.Y. et al. Induction of interleukin-9-producing mucosal mast cells promotes susceptibility to IgE-mediated experimental food allergy. Immunity 43, 788-802 (2015).

38. Stolarski, B., Kurowska-Stolarska, M., Kewin, P., Xu, D. \& Liew, F.Y. IL-33 exacerbates eosinophil-mediated airway inflammation. J. Immunol. 185, 3472-3480 (2010).

39. Komai-Koma, M. et al. Interleukin-33 amplifies IgE synthesis and triggers mast cell degranulation via interleukin-4 in naive mice. Allergy 67, 1118-1126 (2012).

40. Taylor, B.C. et al. TSLP regulates intestinal immunity and inflammation in mouse models of helminth infection and colitis. J. Exp. Med. 206, 655-667 (2009).

41. Ganeshan, K. et al. Impairing oral tolerance promotes allergy and anaphylaxis: a new murine food allergy model. J. Allergy Clin. Immunol. 123, 231-238 e234 (2009).

42. Muto, T. et al. The role of basophils and proallergic cytokines, TSLP and IL-33, in cutaneously sensitized food allergy. Int. Immunol. 26, 539-549 (2014).

43. Han, H. et al. Thymic stromal lymphopoietin amplifies the differentiation of alternatively activated macrophages. J. Immunol. 190, 904-912 (2013).

44. Bryce, P.J. et al. The $\mathrm{H} 1$ histamine receptor regulates allergic lung responses. J. Clin. Invest. 116, 1624-1632 (2006). 